\title{
Ultrasonic Energy Transmission and Conversion Using a 2-D MEMS Resonator
}

\author{
Y. Zhu, S. O. R. Moheimani, Senior Member, IEEE, and M. R. Yuce, Member, IEEE
}

\begin{abstract}
This letter reports a novel ultrasonic-based method to power biosensors. Compared with commonly used radiofrequency radiation methods, ultrasonic power transmission is relatively safe for the human body and does not cause electronic interference. To extract ambient kinetic energy with arbitrary motion directions, a novel 2-D microelectromechanical systems energy converter is designed with resonance frequencies of $\mathbf{3 8 5 2 0}$ and $38725 \mathrm{~Hz}$. Working in the diagonal direction, the device has a bandwidth of $302 \mathrm{~Hz}$, which is twice wider than that of a comparable 1-D resonator device. A storage capacitor is charged up to $0.95 \mathrm{~V}$ in $15 \mathrm{~s}$, when the converter is driven by an ultrasonic transducer at a distance of $0.5 \mathrm{~cm}$, indicating the energy-conversion capability of $21.4 \mathrm{nW}$.
\end{abstract}

Index Terms-Energy converter, implantable biosensor, microelectromechanical systems (MEMS), ultrasonic transmission.

\section{INTRODUCTION}

$\mathbf{I}$ MPLANTABLE devices and low-power biosensors used for identification, monitoring, and treatment of patients are critical to future healthcare industries. Supplying energy continuously to a biosensor microsystem is a key requirement for its continuous and reliable operation. Radio-frequency (RF) wireless powering and telemetry from outside the human body to an implanted medical device have been shown to enable its uninterrupted operation without the need for a battery replacement [1]. However, the inductive coupling method can cause electromagnetic coupling (EMC), which may interfere with other electronic devices in medical environment [2]. Also, exposing the patient to RF radiation may be unsafe for living tissues [3]. Alternatively, the ultrasound is free from the EMC and is believed to be relatively safe for human's health [4], making ultrasonic wireless power transmission a promising candidate for future implantable devices.

In a typical power harvester, kinetic energy is often converted into electrical energy using electromagnetic, piezoelectric, or electrostatic mechanisms [5]. However, the efficiency of vibration-to-electricity conversion is quite low in existing microgenerators because of the following reasons: 1) Most of

Manuscript received November 13, 2009. First published February 25, 2010; current version published March 24, 2010. This work was supported by Australian Research Council Discovery Grant DP0774287. The review of this letter was arranged by Editor J. Cai.

The authors are with the School of Electrical Engineering and Computer Science, The University of Newcastle, Callaghan, N.S.W. 2308, Australia (e-mail: yongyan.zhu@gmail.com; reza.moheimani@newcastle.edu. au; mehmet.yuce@newcastle.edu.au).

Color versions of one or more of the figures in this letter are available online at http://ieeexplore.iee.org.

Digital Object Identifier 10.1109/LED.2010.2040575

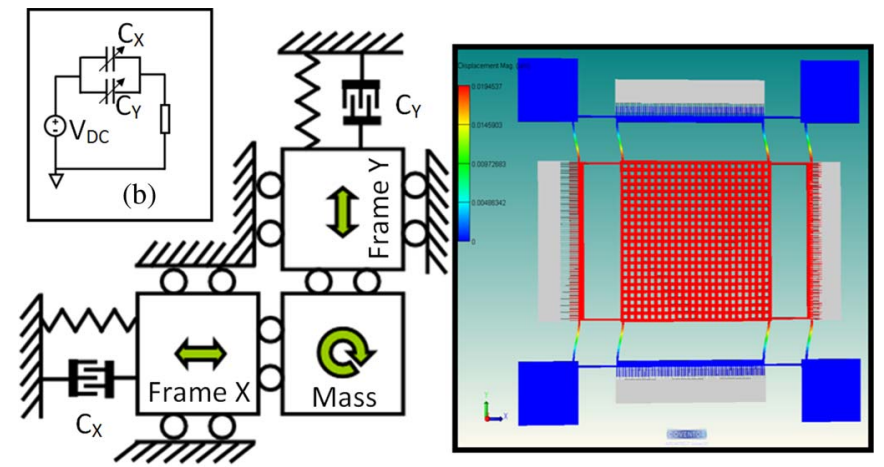

(a)

(c)

Fig. 1. (a) Schematic illustration of the 2-DOF motion mechanism harvesting arbitrary direction vibration. (b) Equivalent circuit model. (c) Graded contour plot of the $X$ mode simulated using CoventorWare.

the reported kinetic generator techniques suffer from scavenging in a narrow bandwidth from ambient sources due to their single resonance frequency, and 2) most devices are designed to harvest energy in a single direction, which wastes the vibration energy existing in other directions [6]. An exception is the multifrequency piezoelectric converter reported in [7], which was shown to broaden the bandwidth by using three piezoelectric bimorph cantilevers with identical dimensions but different masses. Recently, Bartsch et al. reported a 2-D electrostatic resonant micro-energy generator to extract energy from ambient vibrations with arbitrary motion directions. However, the level of harvested power was rather low (100 pW) [8].

In this letter, a proof-of-concept ultrasonic energy transmission method is introduced. A novel two degree-of-freedom (2-DOF) microelectromechanical systems (MEMS) device that vibrates due to incoming ultrasonic waves is designed, fabricated, and tested. The device can scavenge energy in arbitrary directions in a plane and broaden the bandwidth with two resonance frequency peaks. A storage capacitor is charged up to $0.95 \mathrm{~V}$ within $15 \mathrm{~s}$ in a sample experimental test conducted. The power absorption capability of this energy converter is $21.4 \mathrm{nW}$, which is significantly higher than $100 \mathrm{pW}$ of the 2-D electrostatic power harvester reported in [8].

\section{DESIGn ANd FABricAtion}

To harvest vibration energy in arbitrary directions in a plane, 2-DOF motion mechanism has been incorporated in our design, as shown in Fig. 1(a). The seismic mass moves in the $X$ - and $Y$-axes to absorb vibration energy in any in-plane direction. Electrostatic comb variable capacitors $C_{X}$ and $C_{Y}$ collect the $X$ and $Y$ vibration contributions separately and allow the 


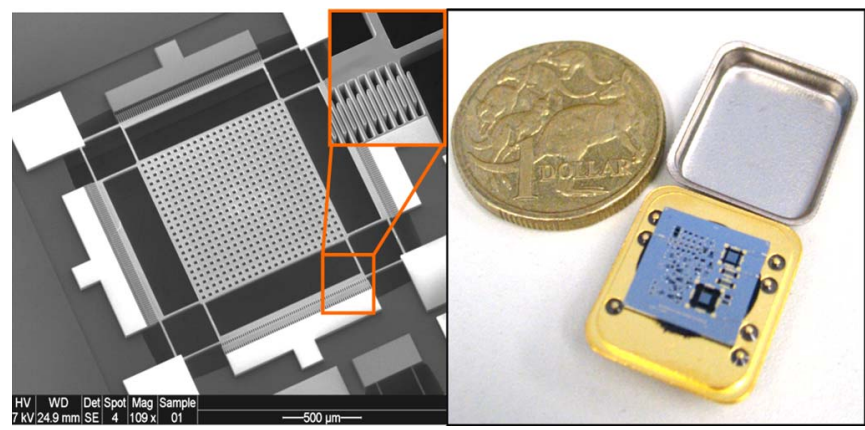

Fig. 2. (a) SEM image of the fabricated energy converter. (b) Photograph of the packaged device.

currents to flow into the load, as shown in Fig 1(b). The capacitors are polarized by a $100-\mathrm{V}$ dc-voltage source. In a practical application, the polarization can be achieved using permanently charged electrets, which remove the need for an external bias voltage [9], [10].

The detailed structure design is shown in Fig. 1(c). The central seismic mass is suspended by 16 beams attached to four corner anchors. The specific topology of the beams splits a certain direction of seismic mass movement into the comb capacitance changing in the $X$ and $Y$ frames. There are two primary resonance frequencies (one for each mode). All 16 beams were designed to have identical dimensions. However, the masses of the $X$ and $Y$ movable comb fingers were separately adjusted to obtain the requisite resonance frequencies. To broaden the bandwidth, the masses were designed to have different dimensions along the $X$-and $Y$-axes (the width of the $X$ frame is $1.5 \mu \mathrm{m}$ wider than that of the $Y$ frame). A CoventorWare modal analysis was carried out on the mechanical structure, which determined the primary resonance frequency at $39238 \mathrm{~Hz}$ [ $X$ mode, as shown in Fig. 1(c)] and the secondary resonance frequency at $39266 \mathrm{~Hz}$ ( $Y$ mode). Simulation results indicate that, in either mode, the central mass and combs only move in one single axis, showing good decoupling.

The device was fabricated in a commercial silicon-oninsulator MEMS foundry (MEMSCAP) with a $25-\mu \mathrm{m}$-thick device layer and a minimum gap of $2 \mu \mathrm{m}$. The image of the packaged device and a section of it taken under a scanning electron microscope (SEM) are shown in Fig. 2(a). The chip was stuck mechanically on a dual-in-line package using a conductive silver tape and was connected electrically to the pins of the package by gold bonding wires, as shown in Fig. 2(b). The package was soldered on a printed circuit board and fixed on a table for testing.

\section{EXPERIMENTAL RESUlTS AND DisCUSSION}

To validate the design in terms of energy conversion, a proof-of-concept measurement has been performed using the fabricated device. The measurement setup is shown in the inset of Fig. 3. An ultrasonic transducer (SQ-40T) was utilized to generate ultrasonic waves. This transducer has a resonance frequency of around $40 \mathrm{kHz}$, which is conveniently close to the resonance frequencies of the designed 2-D resonator. Thus, high-energy coupling is expected to be achieved between the

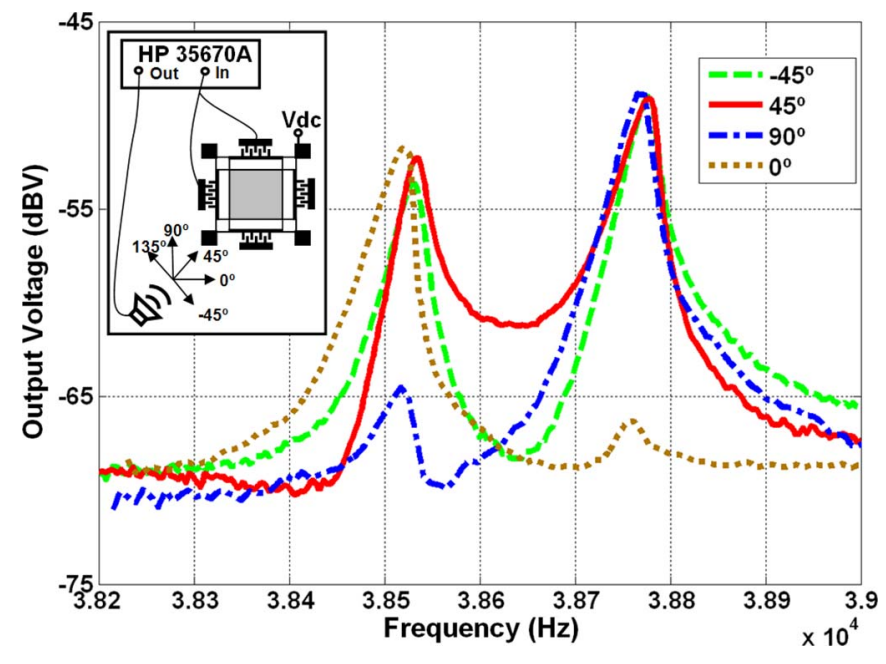

Fig. 3. Magnitude curves of the output voltage versus frequency.

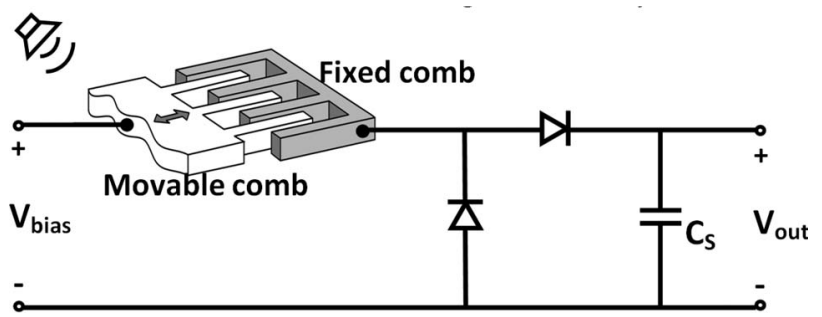

Fig. 4. Energy-conversion circuit diagram.

ultrasonic transducer and the energy converter. The seismic mass absorbs the ultrasonic energy and vibrates in the $X-Y$ plane. While the mass is moving, the comb capacitance is changing also. As the comb capacitors are polarized by a dc-voltage source, the moving combs force the currents into the input node of the signal analyzer.

Fig. 3 shows the obtained frequency response of the converter, with the ultrasonic transducer aiming at varied directions, as shown in the inset. With the transducer having an angle of $0^{\circ}$ with the $X$-axis, one resonance frequency peak is observed, located at around $38.52 \mathrm{kHz}$. The small peak exists at $38.75 \mathrm{kHz}$, which is caused by mechanical cross-axis coupling. The results of the $90^{\circ}$ case are similar to those of the $0^{\circ}$ case. When the direction comes to $45^{\circ}$, two resonance frequency peaks are observed. The $-10-\mathrm{dB}$ bandwidth is $302 \mathrm{~Hz}$, which is twice wider than that of a comparable 1-D resonator. The results of $-45^{\circ}$ and $-135^{\circ}$ directions were the same, having two peaks at 38.52 and $38.75 \mathrm{kHz}$. However, the magnitude between these two peaks is lower than that in the $45^{\circ}$ case because of the antiphase currents contributed by the two connected combs.

Ultrasonic energy is transformed into electrical energy in the form of ac current in Fig. 3. However, rectification of ac-todc voltage must be done prior to powering a biosensor. Fig. 4 shows the ac-dc-voltage rectifier for the energy management. The rectifying bridge circuit consists of two STMicroelectronics 1N5711 small-signal Schottky diodes and a $1-\mu \mathrm{F}$ storage capacitor. These Schottky diodes have low forward-voltage drop of approximately $0.2 \mathrm{~V}$, thereby allowing the largest $\mathrm{dc}$ voltage on storage capacitor $C_{S}$. 


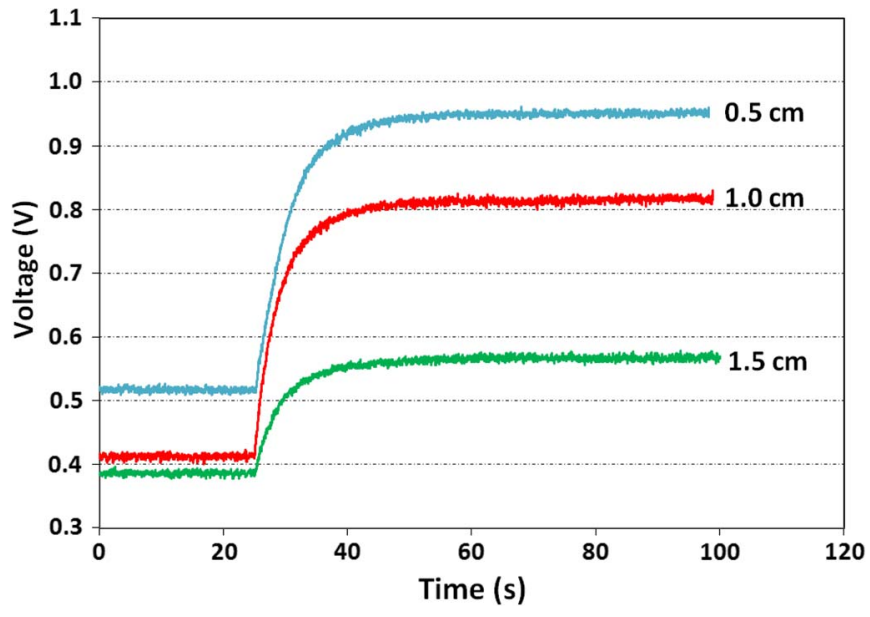

Fig. 5. Voltage profile of $1-\mu \mathrm{F}$ storage capacitor with different gaps $(0.5,1.0$, and $1.5 \mathrm{~cm}$ ) between the ultrasonic transducer and the MEMS energy converter.

Fig. 5 shows the voltage profile of $1-\mu \mathrm{F}$ storage capacitor with different air gaps $(0.5,1.0$, and $1.5 \mathrm{~cm})$ between the ultrasonic transducer and the MEMS energy converter. The bias voltage is $60 \mathrm{~V}$, and the ultrasonic transducer is actuated by $10-\mathrm{V} 38.53-\mathrm{kHz}$ ac voltage, which matches the $X$-mode resonance frequency of the converter. Due to the ultrasonic attenuation through air, the converted voltage on the storage capacitor decreases with the increasing gap. The initial "background" voltage is the feedthrough signal from the ultrasonic actuation ac signal through the stray capacitance between the ultrasonic transducer and the converter. The smaller the gap, the larger the stray capacitance, which corresponds to higher feedthrough signal on the storage capacitor.

After the ultrasonic wave was applied on the converter, the dc voltage on the storage capacitor was charged up from 0.51 to $0.95 \mathrm{~V}$ in $15 \mathrm{~s}$ with $0.5-\mathrm{cm}$ air gap. The power stored in the capacitor is calculated with the formula $P=1 / 2 C V^{2} / T$, where $T$ is the charging time. Thus, the energy stored on the storage capacitor changed from 0.13 to $0.451 \mu \mathrm{J}$ in $15 \mathrm{~s}$, indicating that the power absorption capability of this energy converter is $21.4 \mathrm{nW}$.

A possible ultrasonic power transmission scheme is shown in Fig. 6. An ultrasonic transducer is placed on the patient's skin, in proximity to the biosensor. The ultrasonic energy can be coupled to the patient's body quite efficiently, using a specialized gel (for example, Aquasonic 100 [11]). A MEMS device (i.e., energy generator) inside the body absorbs the ultrasonic energy and transforms it into an electrical charge that is then stored in a rechargeable battery, after diode rectification.

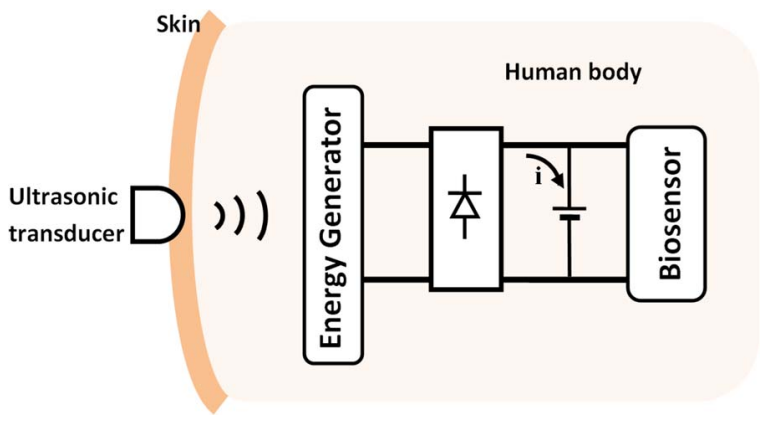

Fig. 6. Schematic diagram of an ultrasonic wireless power supply system.

\section{CONCLUSION}

The modeling, fabrication, and characterization of an ultrasonic-based transducer used for energy conversion have been introduced. The 2-D resonator can extract ultrasonic energy from all directions in the device plane and broaden the bandwidth, increasing the efficiency of energy scavenging. Some 21.4-nW power was converted on a $1-\mu \mathrm{F}$ storage capacitor by wireless energy transmission in an air gap of $0.5 \mathrm{~cm}$. Ultrasonic energy transmission through living tissues will be investigated in future research.

\section{REFERENCES}

[1] M. D. Zimmerman, N. Chaimonart, and D. J. Young, "In vivo powering for advanced biological research," in Proc. 28th IEEE EMBS Annu. Int. Conf., New York, 2006, pp. 2506-2509.

[2] S. Arra, J. Leskinen, J. Heikkila, and J. Vanhala, "Ultrasonic power and data link for wireless implantable applications," in Proc. Int. Symp. Wireless Pervasive Comput., 2007, pp. 567-571.

[3] S. S. Saliterman, Fundamentals of BioMEMS and Medical Microdevices. New York: Wiley-Interscience, 2006, ch. 14.

[4] J. Machi and E. D. Staren, Ultrasound for Surgeons. Baltimore, MD: Williams \& Wilkins, 2004.

[5] U. Bartsch, A. Trautmann, P. Ruther, J. Gaspar, and O. Paul, "Electrostatic transducers for micro energy harvesting based on SOI technology," in Proc. Transducers, 2007, pp. 141-144.

[6] M. Marzencki, Y. Ammar, and S. Basrour, "Integrated power harvesting system including a MEMS generator and a power management circuit," Sens. Actuators A, Phys., vol. 145/146, pp. 363-370, Jul./Aug. 2008.

[7] M. Ferrari, V. Ferrari, M. Guizzetti, D. Marioli, and A. Taroni, "Piezoelectric multifrequency energy converter for power harvesting in autonomous microsystems," Sens. Actuators A, Phys., vol. 142, no. 1, pp. 329-335, Mar. 2008.

[8] U. Bartsch, J. Gaspar, and O. Paul, "A 2D electret-based resonant micro energy harvester," in Proc. IEEE MEMS, Sorrento, Italy, Jan. 25-29, 2009, pp. 1043-1046.

[9] T. Sterken, P. Fiorini, G. Altena, C. Van Hoof, and R. Puers, "Harvesting energy from vibrations by a micromachined electret generator," in Proc. Transducers, 2007, pp. 129-132.

[10] F. Peano and T. Tambosso, "Design and optimization of a MEMS electretbased capacitive energy scavenger," J. Microelectromech. Syst., vol. 14, no. 3, pp. 429-435, Jun. 2005.

[11] [Online]. Available: http://www.parkerlabs.com/aquasonic100.html 